\title{
CRESCIMENTO DO CAMARÃO-SETE-BARBAS (XIPHOPENAEUS KROYERI (HELLER 1862)), NA BAÍA DE TIJUCAS, TIJUCAS, SC (BRASIL).
}

\author{
BRUNO RIBEIRO DE CAMPOS ${ }^{1}$, JOAQUIM OLINTO BRANCO ${ }^{3}$ \& FERNANDO D'INCAO ${ }^{2}$ \\ ${ }^{1}$ Programa de Pós-graduação em Aqüicultura, Instituto de Oceanografia, Universidade Federal do Rio Grande, Avenida Itália, Km 8, $96201-900$ \\ Rio Grande, RS, Brasil. brcampos@yahoo.com \\ ${ }^{2}$ Instituto de Oceanografia, Universidade Federal do Rio Grande, Avenida Itália, Km 8, 96201-900 Rio Grande, RS, Brasil. docdinca@furg.br \\ ${ }^{3}$ Universidade do Vale do Itajaí, Campus I, Centro de Ciência Tecnológicas da Terra e do Mar, UNIVALI, Itajaí, SC, Brasil. branco@univali.br
}

\section{RESUMO}

O camarão-sete-barbas (Xiphopenaeus kroyeri (Heller, 1862)), pertence à superfamília Penaeoidea, família Penaeidae. Apresenta ampla distribuição geográfica no Atlântico Ocidental, ocorrendo da Carolina do Norte (EUA) ao Estado do Rio Grande do Sul. O presente estudo foi motivado pela necessidade de um melhor conhecimento da biologia e do ciclo de vida do camarão-sete-barbas na Baía de Tijucas, Tijucas, Santa Catarina. O crescimento foi avaliado, para ambos os sexos, através da estrutura das distribuições de freqüência de comprimento, utilizando o modelo de BERTALANFFY (1938), o qual foi aplicado nas amostras oriundas da frota artesanal de arrasto. As equações de crescimento obtidas foram: CC $=31\left(1-e^{(-0,0072(t))}\right)$ (fêmeas) e CC = $28\left(1-\mathrm{e}^{(-0,0082(\mathrm{t}))}\right)$ (machos). Os machos apresentaram menor longevidade (561 dias), maior coeficiente de crescimento $(0,0082)$ e menor comprimento assintótico $(28 \mathrm{~mm})$, enquanto as fêmeas crescem mais lentamente $(0,0072)$, vivem por um período mais longo (641 dias) e atingem maiores comprimentos assintóticos ( $31 \mathrm{~mm}$ ).

PALAVRAS CHAVE: Xiphopenaeus kroyeri, crescimento, Tijucas

\section{ABSTRACT}

Growth of sea-bob-shrimp (Xiphopenaeus kroyeri (Heller 1862)), in Tijucas Bay, Tijucas, SC (Brazil). The Sea-bob-shrimp, Xiphopenaeus kroyeri (Heller, 1862), is widely distributed throughout the Western Atlantic, occurring from North Carolina (USA) to Rio Grande do Sul State (Brazil). The study was accomplished in Tijucas Bay, Tijucas, Santa Catarina State with the objective of contributed with the knowledge of biology and life cycle of the species. Samples were obtained from trawl net artisanal fishery. Growth was estimated, for both sexes, through modal progression analysis (MPA), by using the Bertalanffy growth model. The growth equations obtained were: $C L=31\left(1-\mathrm{e}^{(-0.0072(t-0))}\right)$ (female) and $C L=28\left(1-\mathrm{e}^{(-0.0082(t-0))}\right)$ (male). Males presented lower longevity values (561 days), higher growth coefficient $(0.0082)$ and smaller asymptotic length (28 $\mathrm{mm}$ ) than females. Females presented slow growth (0.0072), longevity of 641 days and asymptotic length of $31 \mathrm{~mm}$.

KEYWORDS: Xiphopenaeus kroyeri, growth, Tijucas

\section{INTRODUÇÃO}

A pesca de camarões no litoral brasileiro é realizada em grande escala, com significativa importância econômica, histórica, social e cultural (Branco, 2005). As principais espécies de camarões da plataforma continental das regiões Sudeste e Sul do Brasil são pertencentes à família Penaeidae, destacando-se Xiphopenaeus kroyeri (Heller, 1862) como um importante recurso pesqueiro (D'Incao et al., 2002). Esta espécie apresenta ampla distribuição geográfica no Atlântico Ocidental, ocorrendo da Carolina do Norte (EUA) ao Estado do Rio Grande do Sul (D'Incao, 1999) e não apresenta estratificação populacional, i.e. a ocorrência de juvenis e adultos na mesma área é comum, preferindo águas costeiras rasas com fundo de areia e lama (Iwai, 1973). O ciclo de vida em torno de 18 meses mostra um rápido processo de crescimento (Neiva \& Wise, 1963).

Aspectos biológicos e pesqueiros, bem como o crescimento do camarão-sete-barbas no litoral brasileiro foram estudados no Nordeste (Mota-Alves \& Rodrigues, 1977; Coelho \& Santos, 1993; Santos \&
Coelho, 1996; Santos \& Ivo, 2000), São Paulo (Nakagaki \& Negreiros-Fransozo, 1998; Fransozo et al., 2000; Castro et al., 2005) e Santa Catarina (Nascimento \& Poli, 1985; Branco 2005; Branco \& Verani, 2006) contribuindo com informações relevantes para a espécie.

O estudo do crescimento é ainda importante para a administração dos estoques pesqueiros, contribuindo para obtenção das estimativas de mortalidade e para os modelos de avaliação pesqueira (Garcia \& Le Reste, 1981; Gulland \& Rotschild, 1981; King, 1997). De acordo com D'Incao \& Fonseca (1999) novas estimativas dos parâmetros do modelo de crescimento de Bertalanffy (1938) para peneídeos são necessárias, uma vez que em muitos casos, os parâmetros estimados não condizem com o ciclo de vida das espécies.

Assim, baseado nos antecedentes citados acima, o objetivo do presente trabalho foi estimar a curva de crescimento do camarão-sete-barbas na Baía de Tijucas, Tijucas, SC. 


\section{MATERIAIS E MÉTODOS}

As coletas foram realizadas na Baía de Tijucas (W $48^{\circ} 36^{\prime} 47^{\prime \prime}$ e $S 27^{\circ} 14^{\prime \prime} 46^{\prime \prime}$ ), um dos principais locais da pesca artesanal do camarão-sete-barbas no Estado de Santa Catarina (Fig. 1).

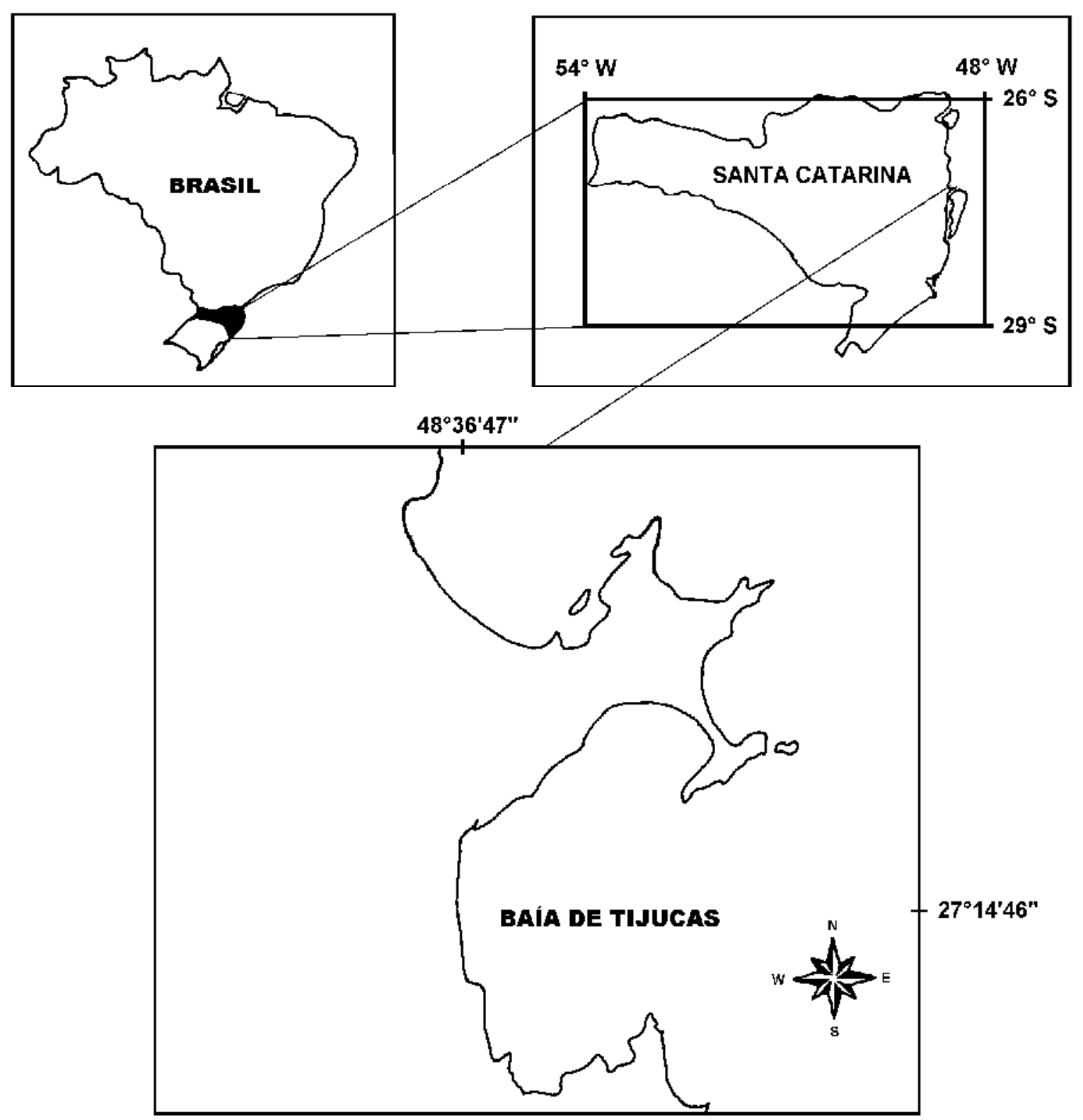

FIGURA 1 - Mapa da região indicando a localização da coleta durante o período de estudo.

As amostragens foram efetuadas a bordo de um barco da frota artesanal, tipo baleeira, de aproximadamente $10 \mathrm{~m}$ de comprimento, $2,5 \mathrm{~m}$ de boca, motor de $30 \mathrm{Hp}$. Foram utilizadas duas redesde-arrastos de portas, malhas (medidas esticadas entre nós opostos) de 3,0 cm (manga e corpo) e 2,0 cm (região do saco). A mesma embarcação foi utilizada ao longo de todo período amostral (janeiro a dezembro de 2005), com freqüência mensal. Em cada saída de campo foi realizado um arrasto, com duração de 1 hora, velocidade de arrasto de 2 nós e na profundidade de $15 \mathrm{~m}$. 
Do total de camarões coletados, foi retirada uma sub-amostra $(1,0 \mathrm{~kg})$ para a realização de biometria. Naquelas em que o peso total foi inferior a esse valor, a amostra foi processada integralmente. Durante o período de estudo, foram medidos 4351 exemplares de X. kroyeri, sendo 1647 (37,85 \%) machos e 2704 $(62,15 \%)$ fêmeas. O comprimento de carapaça (CC ângulo orbital até a porção póstero-dorsal da carapaça) foi medido em milímetros (paquímetro - 0,1 mm de precisão) (Neiva \& Mistakidis, 1966).

Os dados foram separados em classes de 1 $\mathrm{mm}$ de tamanho e originaram distribuições de frequência de comprimentos. As distribuições mostraram o deslocamento das modas ao longo do tempo. Curvas normais foram ajustadas a picos de distribuição. Os picos foram interpretados como grupos etários cujo deslocamento em função do tempo permitiu estimar a curva de crescimento através da análise da progressão modal, utilizando o método de máxima verossimilhança para estimar os parâmetros da curva de Bertalanffy (1938). Para cada um dos ajustes foi calculado o valor de F crítico, com probabilidade de 0,05. Em todos os ajustes o valor de $\mathrm{F}$ esteve acima do $\mathrm{F}$ crítico, significando a sua aceitação para análise. As médias das curvas normais ajustadas foram lançadas em um gráfico de dispersão contra o tempo (dias), para acompanhar o ritmo de crescimento das coortes.

O comprimento assintótico $\left(\mathrm{CC}_{\infty}\right)$ foi fixado de acordo com as médias de comprimentos de carapaça máximos observados nas amostras e na literatura para ambos os sexos (Amado, 1978; Branco et al., 1994; 1999; Nascimento \& Poli, 1985) (Tabela 1).

TABELA 1 - Comprimento de carapaça (CC) do camarão-sete-barbas (Xiphopenaeus kroyeri) citado na literatura e observado no presente estudo.

\begin{tabular}{|c|c|c|c|}
\hline \multirow{2}{*}{ Autores } & \multirow{2}{*}{ Estado } & \multicolumn{2}{|c|}{ Comprimento de Carapaça (mm) } \\
\hline & & Machos & Fêmeas \\
\hline Amado (1978) & PR & & 31,2 \\
\hline Branco et al., (1994) & PR & 26,5 & 31,3 \\
\hline Branco et al., (1999) & SC & & 27 \\
\hline Nascimento \& Poli (1985) & SC & 31,6 & 33,6 \\
\hline Presente estudo & SC & 25 & 31 \\
\hline Média & & 28 & 31 \\
\hline
\end{tabular}

Os parâmetros de crescimento foram estimados para as diferentes coortes de maneira interativa. A partir de valores "semente", foi minimizada a soma dos resíduos entre os comprimentos observados em campo e os calculados pelo modelo de Bertalanffy (1938), variando os parâmetros da equação ( $k$ e $\left.t_{0}\right)$ de maneira interativa. As curvas selecionadas foram aquelas que apresentaram bom coeficiente de determinação $\left(R^{2}\right)$ e

\section{RESULTADOS}

As distribuições de frequência de comprimento de carapaça são polimodais, o que reflete a coexistência de diferentes grupos de tamanho na população (Figura 2). Os meses com maior número de boa correlação com o ciclo de vida da espécie.

A equação da curva de crescimento de Bertalanffy (Bertalanffy, 1938) é como segue:

$$
\mathrm{CCt}=\mathrm{CC}_{\infty}\left[1-\mathrm{e}^{(-\mathrm{k}(\mathrm{t}-\mathrm{t} 0)}\right]
$$

onde CCt é comprimento de carapaça na idade $\mathrm{t}, \mathrm{CC}_{\infty}$ é o comprimento assintótico, $\mathrm{k}$ é a constante de crescimento e $t_{0}$ um parâmetro de ajuste relacionado com o tamanho do indivíduo ao nascer.

modas foram julho (machos - 4 modas), janeiro e abril (fêmeas -5 modas). A incorporação de novas coortes à população ocorre ao longo de todo período amostral, para ambos os sexos, sugerindo recrutamento 
contínuo com picos. Foram ajustadas 9 curvas de crescimento para machos e 14 para fêmeas.

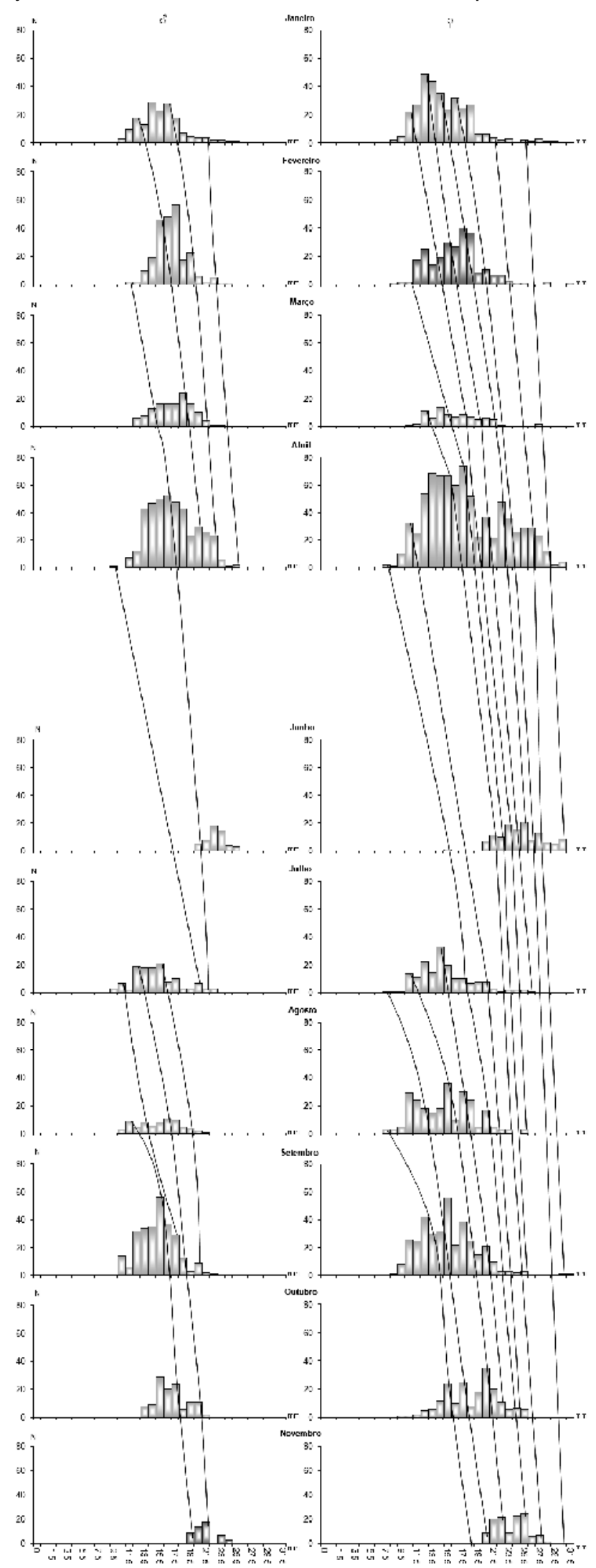

FIGURA 2 - Análise da progressão modal (linhas) de machos (esquerda) e fêmeas (direita) de Xiphopenaeus kroyeri da Baia de Tijucas, SC, durante o período de estudo. 
Os valores fixados de $\mathrm{CC}_{\infty}$, através do ajuste das curvas de crescimento, foram:

$28 \mathrm{~mm}$ (Machos)

$31 \mathrm{~mm}$ (Fêmeas)

O comprimento máximo de carapaça da espécie foi estimado por uma curva de crescimento média que considera a totalidade das curvas estimadas para cada sexo (Fig. 3).

Os parâmetros estimados pelo modelo de Bertalanffy são:

\begin{tabular}{ccc}
\hline & Machos & Fêmeas \\
\hline $\mathrm{k}(\mathrm{dia})$ & 0,0082 & 0,0072 \\
$\mathrm{t}_{0}(\mathrm{dia})$ & 0,0690 & 0,0986 \\
$\mathrm{CC}_{\infty}$ & 28 & 31 \\
Longevidade & 561 dias $(1,54$ anos $)$ & 641 dias $(1,6$ anos $)$ \\
\hline
\end{tabular}
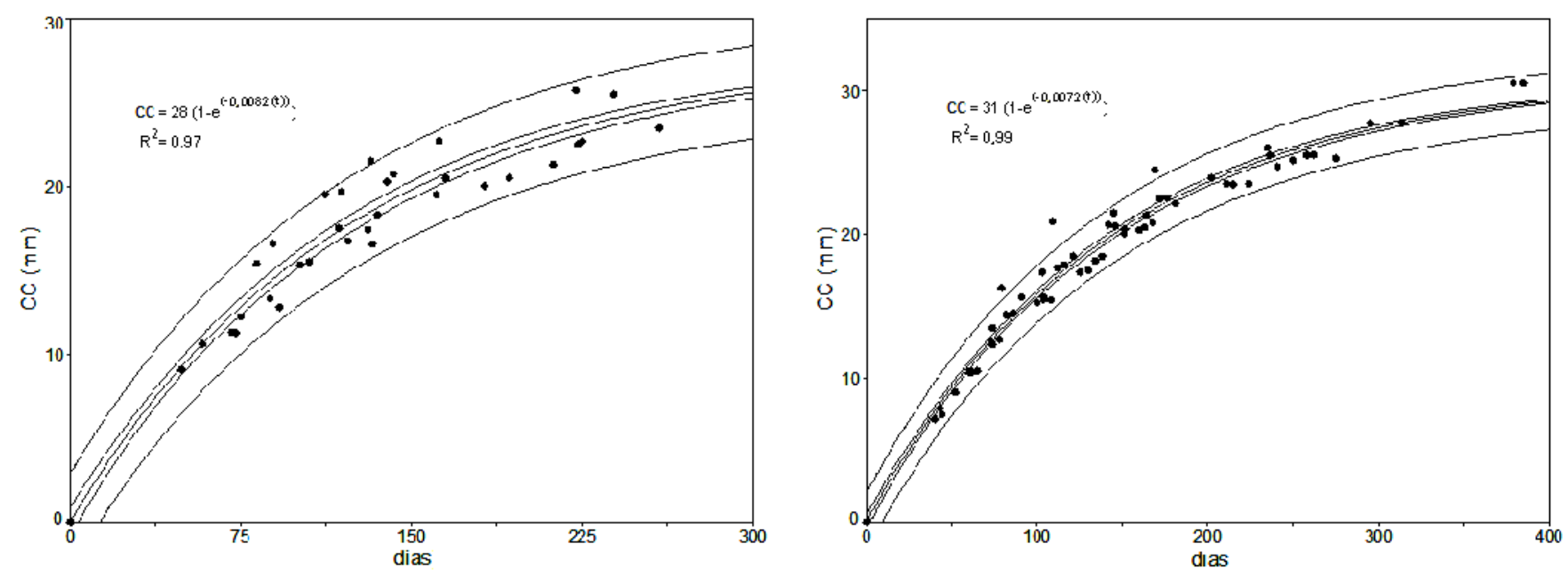

FIGURA 3 - Progressão Modal (CC) correspondendo a diferentes coortes selecionadas para machos e fêmeas de Xiphopenaeus kroyeri na Baía de Tijucas, Tijucas, SC.

A comparação estatística (teste $F$ ) entre as curvas estimadas para machos e fêmeas demonstrou uma diferença significativa entre o $\mathrm{F}$ calculado e o $\mathrm{F}$ crítico (Fcalc $=13,15>$ Fcrít $=3,08$ ) mostrando que as fêmeas crescem de forma mais lenta, mas alcançam maiores tamanhos.

\section{DISCUSSÃo}

Crustáceos não possuem estruturas de aposição que possam ser usadas para se determinar a idade (King, 1997). Desta maneira, a determinação da idade de crustáceos em condições naturais é geralmente feita de forma indireta, utilizando-se o tamanho dos indivíduos como medida para inferir sobre a idade. Dentre os métodos existentes, a análise de progressão modal é o mais comum (Hartnoll, 1982). Considerando este método, é importante avaliar se a curva de crescimento estimada tem coerência biológica, ou seja, que esteja de acordo com as características do ciclo de vida da espécie.
A revisão dos parâmetros de crescimento estimados para peneídeos feita por D'Incao \& Fonseca (1999), mostrou que estimativas de longevidade, considerando $t_{\max }$ como $99 \%$ do comprimento assintótico, geraram valores entre 0,24 e 7,38 anos. As estimativas do coeficiente de crescimento (k), em base anual, variaram entre $0,62 \mathrm{e}$ 18,8 verificando que quanto maior o $\mathrm{k}$ menor a longevidade.

Garcia \& Le Reste (1981) consideram a longevidade máxima dos camarões peneídeos variando entre 2 e 2,5 anos e valores de $k$ entre $1,8 \mathrm{e}$ 3,6. Para Xiphopenaeus kroyeri na Baía de Tijucas, 
foi estimada a longevidade em 561 dias ou 1,54 anos e $\mathrm{k}=0,0082$ (dias) ou 2,99 (anos) para machos e 641 dias ou 1,76 anos e $k=0,0072$ (dias) ou 2,63 (anos) para fêmeas, período este dentro dos limites previamente estabelecidos em outras localidades.

A temperatura da água afeta diretamente o comprimento assintótico $\left(\mathrm{L}_{\infty}\right)$ e a taxa de crescimento (k) (Beverton \& Holt, 1959) e é uma das mais importantes propriedades físicas do ambiente marinho, exercendo influência direta nos eventos químicos, geoquímicos e biológicos. Em peneídeos, o crescimento é fortemente influenciado por fatores biológicos e ambientais (Garcia \& Le Reste, 1981).

Entre as espécies de peneídeos, é comum que os machos apresentem maiores valores de $k \mathrm{e}$, consequentemente, alcancem menores comprimentos assintóticos (Gulland \& Rotschild, 1981; Boschi, 1969).

As estimativas de comprimento assintótico (carapaça) para $X$. kroyeri na região nordeste do Brasil apresenta valores maiores que os citados para a região Sudeste, não estando de acordo com o esperado (Citação). Pauly (1980) considera que águas com temperaturas mais quentes são responsáveis pela aceleração do processo metabólico, resultando em maiores valores de $\mathrm{k}$ e um menor comprimento assintótico. Mas outros fatores podem estar interferindo nessa questão; o esforço de pesca exercido em cada região, pode também influenciar a composição dos tamanhos individuais em populações exploradas. Em um primeiro momento, o comprimento máximo poderia estar diretamente relacionado ao esforço amostral, isto é, com 0 incremento do esforço pesqueiro a probabilidade de capturar indivíduos maiores é maior (D'Incao \& Fonseca, 1999). Entretanto, de acordo com Garcia \& Le Reste (1981), quando o esforço pesqueiro é alto, e com o incremento na intensidade de pesca, uma das conseqüências dessa sobrepesca é o decréscimo no tamanho médio e idade, condição esta bastante aceitável para espécies com interesse comercial.

Durante o período de 1990-1999, a pesca do camarão-sete-barbas no sudeste do Brasil mostrou um decréscimo significativo de $40 \%$ (D'Incao et al., 2002) quando comparado com os dados de pesca obtidos por Valentini et al. (1991) para o período de
1972-1987. Esta tendência de decréscimo no Sudeste do Brasil persistiu nas análises para o período de 1994-2004; o mesmo acontece quando a pesca do estado de Santa Catarina é analisada separadamente (CEPSUL, 2006).

Considerando a situação descrita acima, podese considerar algumas hipóteses para serem testadas no futuro:

- As populações da região Norte e Sul apresentam características distintas de parâmetros morfológicos, podendo assumir a possibilidade de serem diferentes populações.

- O maior valor de $L \infty$ e $k$ na região Norte pode ser relacionado com o aumento do esforço de pesca, aumentando assim a possibilidade de capturar indivíduos maiores.

- Comparando as diferenças entre as populações da região Norte e Sul, em especial em Santa Catarina, podem ser considerados que os valores mais baixos de $L_{\infty}$ e $k$ na região Sul sejam um efeito da sobrepesca, corroborando a análise temporal do estoque estudada por Valentini et al. (1991) e D'Incao et al. (2002).

Analisando a progressão modal e os resultados obtidos, conclui-se que a longevidade obtida é válida por apresentar coerência com o ciclo de vida da espécie. Os comprimentos assintóticos apresentaram coerência com os dados observados em campo para os dois sexos, apoiando as estimativas obtidas para as curvas de crescimento. Na literatura, os valores encontrados de comprimento assintótico variaram de 22,9 a $34,8 \mathrm{~mm}$ para machos e 27,0 a $38,1 \mathrm{~mm}$ para fêmeas; as presentes estimativas $(28 \mathrm{~mm}$ - machos; $31 \mathrm{~mm}$ - fêmeas) estão dentro destes limites.

Segundo D'Incao \& Fonseca (1999) o comprimento assintótico não deve ser considerado só como um valor matemático, sendo que estimativas válidas para esse parâmetro podem variar em até $10 \%$ do comprimento máximo observado. Os valores de longevidade revelam a coerência das curvas de crescimento obtidas com o ciclo de vida da espécie, e com os comprimentos observados nas amostragens e na literatura, enfatizado pela semelhança entre as estimativas de $L_{\infty}$ e os tamanhos máximos registrados nas amostragens (fêmeas $=31 \mathrm{~mm}$ e machos $=28 \mathrm{~mm}$ ).

A validação da curva de crescimento em 
crustáceos usando a longevidade é uma metodologia que favorece a obtenção de estimativas mais coerentes às características do ciclo de vida da espécie e proporcionam uma melhor identificação de eventuais distorções nas estimativas dos parâmetros.

\section{AGRADECIMENTOS}

Ao Projeto Pesca Responsável na Baía de Tijucas, SC, apresentado pela UNIVALI e financiado pelo Fundo Nacional de Meio Ambiente e Coordenado pelo Prof. MSc. Roberto Wahrlich.

\section{REFERÊNCIAS}

AMADO, MAPM. 1978. Estudo biológico do Xiphopenaeus kroyeri (Heller, 1862) (Crustacea, Penaeidae) de Matinhos, Paraná. Dissertação de Mestrado, Curitiba, Universidade Federal do Paraná, 94p.

BERTALANFFY, L VON. 1938. A quantitative theory of organic growth (Inquities on growth laws II). Human Biology, 10(2): 181213.

BEVERTON, RJH \& SJ HOLT. 1959. A review of the lifespans and mortality rates of fish in nature and their relations to growth and others physiological characteristics. In: OLSTENHOLME, GEW \& M O'CONNOR. Eds. The lifespan of animals. London, CIBA Foundation colloquium on ageing, (5). 142-177.

BOSCHI, EE. 1969. Estudio biológico pesquero del camarón Artemesia longinaris Bate, de Mar del Plata. Boletin Biologia Marina, 18: 1-47.

BRANCO, JO, MJ LUNARDON-BRANCO \& A DE FINIS. 1994. Crescimento de Xiphopenaeus kroyeri (Heller, 1862) (Crustacea, Natantia, Penaeidae) da região de Matinhos, Paraná, Brasil. Arquivos de Biologia e Tecnologia, Curitiba, 37(1): 1-8.

BRANCO, JO, MJ LUNARDON-BRANCO, FX SOUTO \& CR GUERRA. 1999. Estrutura populacional do camarão setebarbas Xiphopenaeus kroyeri (Heller, 1862), na foz do rio ItajaíAçú, Itajaí, SC, Brasil. Brazilian Archives of Biology and Tecnology, 42(1): 115-126.

BRANCO, JO 2005. Biologia e pesca do camarão-sete-barbas Xiphopenaeus kroyeri (Heller) (Crustacea, Penaeidae), na Armação do Itapocoroy, Penha, SC. Revista Brasileira de Zoologia, 22(4): 1050-1062.

BRANCO, JO \& JR VERANI. 2006. Pesca do camarão sete-barbas e sua fauna acompanhante, na Armação do Itapocoroy, Penha, SC. In: BRANCO, JO \& AW MARENZI, (Org.). Bases ecológicas para um desenvolvimento sustentável: estudos de caso em Penha, SC. 291. Editora da UNIVALI, Itajaí, SC. p. 153-170

CASTRO, RH, RC COSTA, A FRANSOZO, FL MANTELATTO. 2005. Population structure of seabob shrimp Xiphopenaeus kroyeri (Heller, 1862) (Crustacea: Penaeoidea) in the littoral of São Paulo, Brazil. Sci Mar, 69(1): 105-112

CEPSUL/IBAMA. 2006. Relatório da Reunião Técnica de Análise da Pescaria do Camarão-sete-barbas (Xiphopenaeus kroyeri (Heller, 1862)), 13p.

COELHO, PA \& MCF SANTOS. 1993. Época da reprodução do camarão-sete-barbas, Xiphopenaeus kroyeri (Heller, 1862) (Crustacea, Decapoda, Penaeidae) na região de Tamandaré, PE. Boletim Técnico Científico CEPENE, 1(1): 171-186.

D'INCAO, F 1999. Subordem DENDROBRANCHIATA (camarões marinhos). In: Os crustáceos do Rio Grande do Sul. Ed. Universidade/UFRGS, Porto Alegre: 275-299.

D'INCAO, F \& DB FONSECA 1999. Performance of the von Bertalanffy growth curve in penaeid shrimps: a critical approach. In: The biodiversity crisis and Crustacea. ed. Crustacean Issues Rotterdam, 733-737.

D'INCAO, F, H VALENTINI \& LF RODRIGUES. 2002. Avaliação da pesca de camarões nas regiões Sudeste e Sul do Brasil. 19651999. Atlântica, 24(2): 103-116.

FRANSOZO, A, RC COSTA, MAA PINHEIRO, S SANTOS \& FLM MANTELATTO 2000. Juvenile recruitment of the seabob Xiphopenaeus kroyeri in Fortaleza Bay, Ubatuba, SP, Brazil. Nauplius, 8(2): 179 - 184

GARCIA, S \& L LE RESTE. 1981. Lyfe cycles, dynamics, explotation and management of coastal penaeid shrimp stocks. FAO Fisheries Technical Paper, 203: 1-215.

GULLAND, JA \& BJ ROTHSCHILD 1981. Penaeid shrimps: their biology and management. Fishing News Books. Farnham. Surrey. 308p.

HARTNOLL, RG. 1982. Growth. In: The biology of Crustacea. Embriology, morphology and genetics. Eds. Academic Press: 111-196.

IWAI, M 1973. Pesca exploratória e estudo biológico sobre o camarão na costa Centro/Sul do Brasil com o Navio Oceanográfico "Prof. W. Besnard" em 1969-1971. SUDELPA / IOUSP, São Paulo, 71p.

KING, MG 1997. Fisheries biology, assesment and management. Fishing news books. Osney Mead, Oxford, 341p.

MOTA-ALVES, MI \& MM RODRIGUES. 1977. Aspectos da reprodução do camarão-sete-barbas Xiphopenaeus kroyeri (Heller) (Decapoda, Macrura), na costa do Estado do Ceará. Arquivo de Ciência Marinha, 17(1): 29-35.

NAKAGAKI, JM \& ML NEGREIROS-FRANSOZO. 1998. Population biology of Xiphopenaeus kroyeri from Ubatuba Bay, São Paulo, Brazil. J. Shelf. Res., 17(4): 931-935

NASCIMENTO, PAM \& CR POLI. 1985. Curva de crescimento do camarão-sete-barbas, Xiphopenaeus kroyeri (Heller, 1862), na Baía de Tijucas, Santa Catarina. Anais do I Seminário sobre Ciências do Mar, 1: 37-41.

NEIVA, GS \& JP WISE. 1963. The biology and fishery of the sea bob shrimp of Santos Bay, Brazil. Proceedings of the Gulf and Caribbean Fisheries Institute, 16: 131-139.

NEIVA, GS \& M MISTAKIDIS. 1966. Identificacion de algunos camarones marinos del litoral centro-sur del Brasil. Documentos Tecnicos, CARPAS, Montevideo, 4: 1-10.

PAULY, D. 1980. On the interrelationships between natural mortality, growth parameters, and mean environmental temperature in 175 fish stocks. J. Cons. Int. Explor. Mer. 39(2): 175-192.

SANTOS, MCF \& PA COELHO. 1996. Estudo sobre Xiphopenaeus kroyeri (Heller, 1862) (Crustacea, Decapoda, Penaeidae) em Luis Correia, PI. Trabalhos Oceanográficos da Universidade Federal de Pernambuco, 24: 241-248.

SANTOS, MCF \& CTC IVO. 2000. Pesca, biologia e dinâmica populacional do camarão-sete-barbas, Xiphopenaeus kroyeri 
BRUNO RIBEIRO DE CAMPOS, JOAQUIM OLINTO BRANCO \& FERNANDO D'INCAO

(Heller, 1862) (Crustacea: Decapoda: Penaeidae), capturado em frente ao município de Caravelas (Bahia- Brasil). Boletim Técnico Científico CEPENE, 8(1): 131-164.

VALENTINI, H, F D'INCAO, LF RODRIGUES, JE REBELO-NETO
\& E RAHN. 1991. Análise da pesca do camarão-rosa (Penaeus paulensis e Penaeus brasiliensis) nas regiões sudeste e sul do Brasil. Atlântica, 13(1): 143-157.

Submetido: 04/03/2010

Aceito: $07 / 12 / 2011$ 